Onagrarieae.

Epilobium angustifolium L. Häufig bis an die Alpen. Letze und Illufer bei Feldkirch, Saminathal u. s. w. 1. 2. ***

E. Fleischeri Hochst. Im Montafon und Gargellenthal massenhaft; vereinzelt und wahrscheinlich angeschwemmt am Illdamm zwischen Feldkirch und Nofels; Fuss des Schwarznorn und Sporer Thal. 1. 2.*

E. parviflorum Schreb. Häufig in den Niederungen des unteren Gebietes; Hlufer unterhalb Feldkirch, Tisis u. s. w. 1. **

E. montanum L. Gemein bis in die unteren Alpen, wo nicht selten die var. collinum. 1. 2. **

E. roseum Schrk. Mit E. montanum die gemeinste Art. 1. **

E. trigonum Schrk. Gampertonthal, Arlberg und sonst durch das ganze Gebiet zerstreut. 2. 3. *

E. origanifolium Lam. Gamperthonthal. 3. *

Oenothera biennis L. Im Illsande unterhalb Feldkirch und am Rheinufer. 1.

Circaea lutetiana L. Häufig bis in die unteren Alpen, Ardetzenberg, Margarethenkopf, Steinwald, Saminathal etc. 1. **

Lythrarieae.

Lythrum salicaria L. Gemein um Feldkirch. 1. **

(Fortsetzung folgt.)

\title{
F. Schultz und F. Winter, Herbarium normale.
}

Besprochen von R. v. Uechtritz.

Wohl den ersten Rang in ganz Europa nimmt unter den Herausgebern von getrockneten Pflanzensammlungen heute F. Schultz ein, der seit dem Jahre 1836, wo er die Edition seiner Flora Galliae et Germaniae exsiccata begann, den grissten Theil seines Lebens darauf verwendet hat, durch Herstellung von Exsiccatensammlungen ersten Ranges der Wissenschaft zu nützen. Zur Beendigung jenes ersten bis auf 16 Centurien ausgedehnten Unternehmens wurde der Herausgeber durch den Verlust eines grossen Theiles seiner Vorräthe gezwungen, den er im Jahre 1853 bei Gelegenheit des Umzugs von Bilsch nach seinem gegenwärtigen Domizil Weissenburg durch einen Orkan erlitt. Aber dieses Nalheur war nicht im Stande, unsern Freund Schultz zu entmulhigen; im Gegentheil wurde nunmehr eine grössere auf die Flora von ganz Europa ausgedehnte Sammlung, das He barium normale, in Angriff genommen, von dem bis zum Jahre 186912 Centurien erschienen. Bei seinem vorgeriichten Alter und den grossen Muihseligkeiten, welche der mechanische Theil des Unternehmens, die Besorgung der ausgedehnten Correspondenz und die Vertheilung der Vorrähe erfordert, sah sich Schultz genüthigt, seitdem einen Mit- 
arbeiter in der Person des inzwischen ebenfalls in Weissenburg heimisch gewordenen Bryologen F. Winter anzunehmen, und so erschienen im Jahre 1872 die ersten 3 Centurien der neuen Serie des Herbarium normale, 2 Cent. Phanerogamen, eine Kryptogamen enthaltend, unter gemeinschaftlicher Firma. Da diese schöne Sammlung, welche einzig in ihrer Art dasteht und allen ähnlichen, mitunter vorzugsweise auf Füllung des Geldbeutels der Herausgeber und nur nebenbei auf Förderung der Wissenschaft berechneten Unternehmen den Vorrang streitig macht, verhältnissmässig in Deutschland nicht nach Verdienst bekannt ist, so hält Ref. es für angemessen, auf dieselbe von Neuem aufmerksam zu machen und zu diesem Zwecke die zuletzt erschienenen Centurien zu besprechen.

Vorher noch einige allgemeine Bemerkungen über die Einrichtung der Kollektion im Allgemeinen. Durchschnittich alle 2-3 Jahre erscheint eine Doppelcenturie, fast durchaus nur seltenere oder kritische Arten der europäischen Flora enthaltend, gemeine werden nur vereinzelt des Vergleiches halber ausgegeben. Die Pflanzen liegen frei auf Bogen weissen Papiers vom grössten Format; jeder Art ist eine Etikette beigegeben, welche ausser dem Namen und der mit wissenschaftlicher Gründlichk eit redigirten Synonymik den speziellen Standort und den Namen des Sammlers, sowie meist auch Angaben über das Substrat und die Höhe der Lokalität, nebst dem genauen Datum der Einsammlung enthält. - Die Exemplare sind fast durchwegs reichlich und möglichst vollständig gegeben; in der Mehrzahl der Falle finden wir Blïthen- und Fruchtexemplare zugleich geliefert, selbst bei schwierig zu erlangenden Arten. Varietaten einer Art werden nicht als besondere Nummern gerechnet; nicht wenige Arten sind von mehr als einem Standort ausgegeben und in solchen Fallen werden die Doppelnummern ebenfalls gratis geliefert. So enthalten z. B. die uns vorliegenden drei letzten Centurien 25 Spezies von 2 Standorten, eine (Cinclidotus fontinaloides P. B.) sogar von 3; ausserdem ist ein 14 Nummern umfassendes Supplement zu bereits in früheren Centurien gelieferten Arten gratis beigegeben, so dass im Ganzen statt 300 Nummern deren 341 geliefert werden! - Der Sammlung liegt ein Exemplar der zugehörigen Nummer der "A rchives de Flore" bei, eines dem internationalen Charakter des Unternehmens gemäss in französischer Spracle erscheinenden Journales, in dem die Diagnosen neuer in der Sammlung ausgegebener Arten, sowie kritische Bemerkungen zu den ubrigen überhaupt enthalten sind; der Herausgeber lässt dasselbe, obwohl er nicht bemittelt ist, auf seine Kosten drucken und vertheilt es gratis an die Subskribenten und Sammler seiner Kollektionen, sowie an hervorragende Kollegen und gelehrte Gesellschaften. Solche Uneigennützigkeit verdient in einer so materiell gesinnten Zeit, wie die unsrige, doppelte Anerkennung! - Die 61 Mitarbeiter an den vorliegenden 3 Centurien, unter denen wir Namen wie Jordan, Requien $\left(t^{\circ}\right)$, Timbal-Lagrave, P. Savi $(t)$, J. Lange, Janka, Tommasini elc. begegnen, vertheilen sich nach Nationalitäten wie folgt: 27 Franzosen, 18 Deutsche, 9 aus Oesterreich-Ungarn. 4 
Dänen, ferner je 1 Schwede, Schweizer und Italiener. Sehr schlecht vertreten ist der preussische Staat, indem nur Schlesien und der südliche Theil der Rheinprovinz Rekruten geliefert haben. Auch aus den österreichischen Ländern wäre eine stärkere Betheiligung wünschenswerth. Für diejenigen, welche schon geneigt sein sollten, für Schultz zu sammeln, bemerken wir, dass derselbe für je 10 in 120 instruktiven Exemplare gelieferto Arten ein Exemplar der betreffenden Centurie gibt. - Nun noch einige spezielle Bemerkungen zu den diesmal ausgegebenen Pflanzen.

Das von Kaiserslautern ausgegebene Thalictrum sylvaticum ist von der nordostdeutschen Pflanze, welche einen abweichenden Blïthenstand, grössere freudig grüne Blättchen mit normal slumpferen Lappen und einen ganz abweichenden Habitus besitzt, unseres Bedünkens nach verschieden; auch der auf der Etikette angegebene Standort (Clairières, bruyères et bords des forets de pins) entspricht nicht dem der hiesigen Form, die ausschliesslich in fruchtbaren Laubgehölzen gefunden wird. - Als Batrachium Langei F. Schz. wird provisorisch ein dem Herausgeber von Prof. Lange als $B$. confusum aus Dänemark geschickter Wasserhahmenfuss bezeichnet, der uns indessen identisch mit B. triphyllos Wallr. (Linn. 1840) scheint. Auch wir haben diese Pflanze, als sie uns zuerst aus Schlesien bekannt wurde, irrig für B. confusum gehalten, welches nur in salzhaltigen Gewässern vorzukommen scheint. - Bei Linum perenne L. (ex p.) Koch von Darmstadt wäre das Synonym $\boldsymbol{L}$. darmstadinum Alefeld zu erwähnen gewesen. - Bei Arenaria tetraquetra L., die von zwei Standorten ausgegeben wird, ist zu bemerkeu, dass die Pflanze der Provence zur typischen Form ( $\alpha$ legitima Gr. et G.; $\beta$ aggregata Gay), in tieferen Gegenden vorkommend, gehört, während die von der Peña Blanca in den spanischen Pyrenäen die alpine, habituell äusserst unähnliche Varietät ( $\beta$ condensata Gr. et G.; a uniflora Gay) darstellt. Dies wäre anf den Etiketten anzugeben gewesen, da beide Formen wie gesagt habituell sich viel weniger gleichen, als oft wirklich verschiedene verwandte Arten. Hieracium poliotrichum (n. 90 et $90^{\text {bis }}$ ) von den Striegauer Bergen in Schlesien und aus der Gegend von Crossen in der angrenzenden Mark ausgegeben, ist gegen Rehmanns Behauptung (cfr. Oest. b. Zeit. XXIII. p. 150) die echte Pflanze dieses Namens. Die Striegauer Pflanze, die ich selbst an Schultz eingesendet und die schon früher durch mich in den Hieraciis europaeis von Fries ausgegeben wurde, ist speziell sogar der Typus dieser Art, die bekanntlich von Fries für das wahre $\boldsymbol{H}$. cymosum L. erklärt wird und die Striegauer Berge sind einer der Originalstandorte Wimmer's. An dieser Lokalitat wie in ganz Schlesien vermuthlich überhaupt findet sich die von Rehmann irrig als $\boldsymbol{H}$. cymosum poliotrichum bezeichnete Pllanze durchaus nicht; diese letztere, welche erst kürzlich von Čelakovský ausführlich beleuchtet wurde, gehört gar nicht in den Formenkreis des $\boldsymbol{H}$. cymosum, sondern, wofern man sie nicht als eigene Art betrachten will, in den des H. praealtum. Da Wimmer diverse Formen unter seinem Hiracium alpinum foliosum 
verstanden hat, so ist bei dem gleichlautenden Synonym des neu aufgestellten $\boldsymbol{H}$. Fritzei F. Schz. das ex parte zuzusetzen. - Bei Nr. 94 (H. valdepilosum Vill.) und 95 (H. picroides Vill.) ist in dem Exemplar des Ref. (und nach brieflicher Mittheilung des Herausgebers auch in anderen) durch ein Versehen Winter's, der die Versendung besorgte, eine Etikettenverwechslung passirt; dieselbe ist leicht auch für Unkundige zu verbessern, indem von ersterer Art in allen Exemplaren der Sammlung nur ein Individuum gegeben wurde, während Nr. 95 überall in 3 schönen Individuen geliefert ist. Letzteres, wie Nr. 94 von Huter aus Tyrol mitgetheilt, wird für ein H. strictum $\times$ albidum erklärt, was uns fraglich scheint; die von F. Schz. ausgegebene Pflanze (das H. Huteri $\mathrm{Hsm}$.) ist, wenn überhaupt hybrid, wohl eher ein $H$. hispidum $\times$ albidum $(=$ Bocconei $\times$ intybaceum $)$ (cft. Bot. Zeit. 1872, p. 191 u. 192). - H. racemosum, von Namiest (Mähren) ausgegeben, ist trotz des Stilus fuligineus nicht die Pflanze von W. K., welche in Koch's Florengebiete kaum vorkommt, sondern H. barbatum Tausch oder das H. racemosum der Deutsch-Oesterreicher, welches neuerlich auch in Schlesien gefunden wurde. H. tenuifolium Host. hält Ref. gegen Kerner für nicht spezifisch verschieden; die von diesem angegebenen Differenzen sind nicht bestündig und finden sich oft nur theilweise vereint. Ob $H$. racemosum W. K., Fries und $\boldsymbol{H}$. barbatum durchgreifend spezifisch verschieden sind, darüber hat Ref. kein eigenes Urtheil, da ihm die erstere Pflanze nur aus einem einzigen von Rochel kultivirten Exemplare bekannt ist, welches mit seinem kahlen, nur etwas schärflichen Stengel, den rigiden, grobgezähnten, fast kahlen Blattern, den verlangerten, untern Aesten des Blüthenstandes und den doppelt kleinen Köpfen allerdings den Eindruck einer besonderen Art macht, doch ist zu bemerken, dass $H$. barbatum ziemlich polymorph ist und namentlich auch in der Grösse der Köpfe zu variiren scheint. Ebenso ist der bei Fries angegebene Unterschied in der Farbe des Griffels in Wirklichkeit, wenigstens getrocknet, nicht vorhanden, denn bei sämmtlichen Exemplaren dieser Art, die wir aus Schlesien, Mïhren, Oberungarn, N. Oesterreich und Steiermark gesehen haben, war der Griffel russbraun, wie ihn Fr. für H. racemosum W. K. vorschreibt. Unter Nr. $109^{\text {bis }}$ ist Cuscuta aurantiaca Req. in Requien'schen Originalen von Corsica ausgegeben. Aber Nr. 109 ist C. racemosa Mart. var. chiliana Engelm. (C. corymbosa Choisy, C. hassiana Pfeiffer), das bekannte, in neueren Zeiten in den meisten Gegenden wieder verschwundene Luzernenunkraut, wahrend C. aurantiaca zu der europäischen Form der C. obtusiflora H. B. Kth. gehört; der Herausgeber hat sich offenbar durch die Flore de France täuschen lassen, in welcher C. aurantiaca als Synonym zu C. corymbosa gedeutet wird. - Von anderen besonders interessanten Sachen, welche in dieser Doppelcenturie ausgegeben sind, nennen wir u. a. Thalictrum macrocarpum Gren; Batrachium Lenormandii F. Schz., Polygala nicaeensis, suwohl in der Form mit blauen Blüthen von Nizza, als in der rosafarbenen ( $P$. corsica Boreau) von Pola in Istrien, Trifolium parviflorum Ehrh. von Montbrison (Dep. Loire), mit der 
östlichen Pflanze durchaus übereinstimmend; T. laevigatum Desf.; Rubus Winteri P. Müller; Potentilla pyrenaica Ram.; Bulliarda aquatica und Vaillantii DC; Sempervivum Pittonii Schott; Umbilicus sedoides DC.; Valeriana simplicifolia Kabath (aus Oberschlesien); Cephalaria centauroides Coult. in der bekleideten Form (Scab. corniculata W. K.) aus Siebenbürgen (Janka); Bidens radiatus Th. in Zwergexemplaren aus Danemark; Senecio divergens F. Schz. von Weissenburg, in Westdeutschland vielleicht bisweilen für $S$. erraticus Bert. genommen, dem er im Blïthenstande und in den Köpfen nicht unähnlich ist, von dem er sich aber im Kraute weit entfernt; Centaurea intybacea Lam., C. Hanryi Jordan; Hieracium graniticum Schz. Bip., näher mit $\boldsymbol{H}$. Schmidtii Tsch. verwandt als mit H. murorum, zu dem es neuerlich Rehmann, wohl mit Unrecht, gezogen hat, vom Originalstandorte Namiest in Mahren; H. bohemicum Fries unter der Bezeichnung $\boldsymbol{H}$. sudeticum Sternberg, die jedenfalls für dieses mit grösserem Rechte angewendet werden kann, als für $H$. pedunculare Tausch, welches Grisebach, Wimmer und Fries (Epicr.) als H. sudeticum haben, da jenes und nicht die letzte Art von Sternberg abgebildet wurde. - Symphitum bulbosum Schimp. von Weissenburg, Digitalis laevigata W. K.; Antirrhinum latifolium DC. und sempervirens L., Veronica nummularia Gouan, Orobanche loricata Rchb., O. Picridis F. Schz.; $O$. caerulescens Steph. von Regensburg, vom selig. Fürnrohr gesammelt; Mentha aquatica $\times$ rotundifolia Boutigny; Teucrium pulverulentum (Jord. el Fourr.), eine von T. flavum nur schwach verschiedene Form aus Südfrankreich, Cyclamen neapolitanum Ten. und C. repandum S. et Sm., beide von Pisa; das sonderbare Polygonum equisetiforme S. et Sm. ans Korsika; Dioscorea pyrenaica Bubani et Bordère, dieser merkwürdige, erst in neueren Zeilen entdeckte, einzige europäische Repräsentant der Gattung; Gagea fibrosa R. Sch. von Constantine (Algier), Juncus atratus Krocker aus der Rheinpfalz, ein merkwürdiges Vorkommen dieser sonst mehr östlichen Art; J. Kochii F. Schz. (J. nigritellus Koch non Don) aus der Pfalz und dem rheinpreussischen Saargebict; Carex Leersii F. Schz.; C. latvigata Sm. von Vire (Dep. Calvados), Avena planiculmis Schrad. aus dem Gesenke, Glyceria nemoralis, welche bisher noch nicht in einer Exsiccatensammlung ausgegeben wurde; Festuca pyrenaica Reuter, sowie überhaript eine Menge seltener Alpen-, Pyrenäen- und südeuropaischer Pflanzen; die lelztern meist aus Südfrankreich. Auch die Kryptogamen-Cenlurie, meist Laubmoose enthaltend, bietet viel des Seltenen, so z. B. Isoëtes echinospora Dur. aus dem Dep. Haute-Vienne; Aspidium aculeatum Sw. verum (von Agen), Asplenium Petrarcae DC; A. lanceolatum Huds. von dem deutschen Standorte bei Weissenburg. Tayloria Rudolphiana Br. eur. etc. - Wir nehmen mit dem Wunsche, dass es dem Herausgeber noch recht lange vergönnt sein möchte, seine Kräfte dem schönen Unt arnehmen zu widmen, von der Kollektion Abschied, welche in keiner öffentlichen und grösseren Privatsammlung fehlen sollte und in Bezug deren wir das Urtheil unseres Freundes Crépin mit ganzem Herzen unterschreiben: Sans 
craindre d'ètre contredit et taxé d'exagération, nous pouvons avancer que la collection éditée par M. Schultz est la plus intéressante, la mieux soignée et la plus scientifique qui ait encore paru: on peut difficilement faire mieux.

\section{Correspondenz.}

Kalksburg în Niederösterreich, am 22. September 1873.

Ich habe eine für Niederösterreich und überhaupt für Oesterreich neue Pflanze gefunden, nimlich Geranium sibiricum, welches ich am 12. August nächst Bruck a. d. Leitha auf niederösterreichischem Gebiet und zwar diesseits der Leitha gesammelt habe. Jenseits der Leitha wurde es schon vor Jahren bei Eisenstadt im Steinschutte des Leithagebirges ron Pawlowshi aufgefunden.

Joh. Wiesbauer S. J.

Linz, am 28. September 1873.

Ich theile Ihnen mit, dass Herr Julius Anton Freih. v. Handel, k. k. Statthaltereirath in Pension, am 26. d. M. in Linz gestorben ist. Sein Herbarium, welches vorzugsweise Pflanzen aus Oberösterreich und Salzburg enthält, widmete er noch bei Lebenszeit dem Vereine für Naturkunde in Oberösterreich zu Linz.

Dr. Rauscher.

Graz, am 8. October 1873.

Mein Sohn Otto, Major im Inft-Reg. Nr. 22, war mehrere Jahre in Ragusa stationirt und hat mir Anfangs März Wurzelstöcke und einige blühende lebende Exemplare einer Mandragora von Straccia in Dalmatien verschafft, die ich für die M. autumnalis Sp. erkannte, dieselbe Pflanze, die in Reichenbach's Flora Tom. 20 Tab. 6 abgebildet ist. Im August d. J. erhielt ich Früchte und lose Samen derselben Pflanze, wovon ich Ihnen einige Paquets zur Vertheilung für Ihre Korrespondenten überschicke.

v. Pittoni.

Langenlois in Nied.-Oesterr., am 18. Oktober 1873.

Was die Vegetationsverhältnisse des Weinstockes in diesem Jahre betrifft, so waren dieselben sehr ungünstig, und sind die Hoffnungen der Weinhauer wenig in Erfüllung gegangen. Auf einen eben so gelinden als feuchten Winter erwartete man einen kräftigen Antrieb der Rebe; allein in vielen Weingärten erfolgte das Gegentheil; zu viele Nüsse hatte viele Reben verdorben, sie entwickelten sich sehr spät und trieben nur Blälter, ohne Früchte anzusetzen. In besseren Lagen zeigten sich im Frühlinge sehr viele Trauben, doch die im April eingetretene Kälte, die sich am 28. zum Froste $\left(-3^{0}\right.$ R.) steigerte, zerstörte den jungen Antrieb fast gänzlich, so dass das heurige Erträgniss der Weingärten bei den meisten Weinhauern nur ein Fünftel, bei vielen nur ein Zehntel eines guten Weinjahres lieferte. Bezüglich der Qualität des heurigen Produktes ist es leicht begreiflich, dass bei dem durch den Frost verspäteten Antriebe des 